\title{
The Mind's Eye Mapped Onto the Brain's Matter
}

\author{
Marlene Behrmann ${ }^{1}$ \\ Department of Psychology, Carnegie Mellon University, Pittsburgh, Pennsylvania
}

\begin{abstract}
Research on visual mental imagery has been fueled recently by the development of new behavioral and neuroscientific techniques. This review focuses on two major topics in light of these developments. The first concerns the extent to which visual mental imagery and visual perception share common psychological and neural mechanisms; although the research findings largely support convergence between these two processes, there are data that qualify the degree of overlap between them. The second issue involves the neural substrate mediating the process of imagery generation. The data suggest a slight lefthemisphere advantage for this process, although there is considerable variability across and within subjects. There also remain many unanswered questions in this field, including what the relationship is between imagery and working memory and what representational differences, if any, exist between imagery and perception.
\end{abstract}

\section{Keywords}

mental imagery; visual perception; cognitive neuroscience

Consider sitting in your office and answering the question "How many windows do you have in your living room?" To decide how to answer, you might construct an internal visual representation of your living room from the stored information you possess about your home, inspect this image so as to locate the windows, and then count them. This type of internal visual representation (or "seeing with the mind's eye"), derived in the absence of retinal stimulation, is known as visual mental imagery, and is thought to be engaged in a range of cognitive tasks including learning, reasoning, problem solving, and language. Although much of the research on mental imagery has been concerned specifically with visual mental imagery, and hence the scope of this review is restricted to this topic, similar internal mental representations exist in the auditory and tactile modalities and in the motor domain.

The past decade has witnessed considerable progress in our understanding of the psychological and neural mechanisms underlying mental imagery. This is particularly dramatic because, in the not-too-distant past, during the heyday of behaviorism, discussions of mental imagery were almost banished from scientific discourse: Given that there was no obvious way of measuring so private an event as a mental image and there was no homunculus available for viewing the pictures in the head even if they did exist, the study of mental imagery fell into disrepute. Indeed, through the 1940s and 1950s, Psychological Abstracts recorded only five references to imagery. The study of mental imagery was revived in the 1970s, through advances such as the experiments of Shepard and colleagues (Shepard \& Cooper,
1982) and of Kosslyn and colleagues (see Kosslyn, 1994), and the dual coding theory of Paivio (1979).

Although a general consensus endorsing the existence of mental imagery began to emerge, it was still not fully accepted as a legitimate cognitive process. Some researchers queried whether subjects were simply carrying out simulations of their internal representations in symbolic, nonvisual ways rather than using a visual, spatially organized code. Other researchers suggested that subjects were simply conforming to the experimenters' expectations and that the data that appeared to support a visualarray format for mental imagery merely reflected the experimenters' belief in this format rather than the true outcome of a mental imagery process (Pylyshyn, 1981). In recent years, powerful behavioral and neuroscientific techniques have largely put these controversies to rest. This review presents some of this recent work.

\section{RELATIONSHIP BETWEEN VISUAL IMAGERY AND VISUAL PERCEPTION}

Perhaps the most hotly debated issue is whether mental imagery exploits the same underlying mechanisms as visual perception. If so, generating a visual mental image might be roughly conceived of as running perception backward. In perception, an external stimulus delivered to the eye activates visual areas of the brain, and is mapped onto a long-term representation that captures some of the critical and invariant properties of the stimulus. During mental imagery, the same long-term representations of the visual appearance of an object are used to activate earlier representations in a top-down fashion through the influence of 
preexisting knowledge. This bidirectional flow of information is mediated by direct connections between higher-level visual areas (more anterior areas dealing with more abstract information) and lower-level visual areas (more posterior areas with representations closer to the input).

\section{Mental Imagery and Perception Involve Spatially Organized Representations}

Rather than being based on propositional or symbolic representations, mental images appear to embody spatial layout and topography, as does visual perception. For example, many experiments have shown that the distance that a subject travels in mental imagery is equivalent to that traveled in perceptual performance (e.g., imagining the distance between New York and Los Angeles vs. looking at a real map to judge the distance). Recent neuroimaging studies have also provided support for the involvement of spatially organized representations in visual imagery (see Kosslyn et al., 1999); for example, when subjects form a high-resolution, depictive mental image, primary and secondary visual areas of the occipital lobe (areas 17 and 18, also known as V1 and V2), which are spatially organized, are activated. ${ }^{2}$ Additionally, when subjects perform imagery, larger images activate relatively more anterior parts of the visual areas of the brain than smaller images, a finding consistent with the known mapping of how visual information from the world is mediated by different areas of primary visual cortex. Moreover, when repetitive transcranial magnetic stimulation ${ }^{3}$ is applied and disrupts the normal function of area 17, response times in both perceptual and imagery tasks increase, further supporting the involvement of primary visual areas

in mental imagery (Kosslyn et al., 1999).

\section{Shared Visual and Imagery Areas Revealed Through Functional Imaging}

Not only early visual areas but also more anterior cortical areas can be activated by imagined stimuli; for example, when subjects imagine previously seen motion stimuli (such as moving dots or rotating gratings), area MT/MST, which is motion sensitive during perception, is activated (Goebel, Khorram-Sefat, Muckli, Hacker, \& Singer, 1998). Color perception and imagery also appear to involve some (but not all) overlapping cortical regions (Howard et al., 1998), and areas of the brain that are selectively activated during the perception of faces or places are also activated during imagery of the same stimuli ( $\mathrm{O}^{\prime}$ Craven \& Kanwisher, in press). Higher-level areas involved in spatial perception, including a bilateral parietooccipital network, are activated during spatial mental imagery, and areas involved in navigation are activated during mental simulation of previously learned routes (Ghaem et al., 1997). As is evident, there is considerable overlap in neural mechanisms implicated in imagery and in perception both at lower and at higher levels of the visual processing pathways.

\section{Neuropsychological Data for Common Systems}

There is also neuropsychological evidence supporting the sharedsystems view. For example, many patients with cortical blindness (i.e., blindness due to damage to primary visual areas of the brain) or with scotomas (blind spots) due to destruction of the occipital lobe have an associated loss of imagery, and many patients with visual agnosia (a deficit in recognizing ob- jects) have parallel imagery deficits. Interestingly, in some of these latter cases, the imagery and perception deficits are both restricted to a particular domain; for example, there are patients who are unable both to perceive and to image only faces and colors, only facial emotions, only spatial relations, only object shapes and colors, or only living things. The equivalence between imagery and perception is also noted in patients who, for example, fail both to report and to image information on the left side of space following damage to the right parietal lobe.

There are, however, also reports of patients who have a selective deficit in either imagery or perception. This segregation of function is consistent with the functional imaging studies showing that roughly two thirds of visual areas of the brain are activated during both imagery and perception (Kosslyn, Thompson, \& Alpert, 1997). That is, selective deficits in imagery or perception may be explained as arising from damage to the nonoverlapping regions. Selective deficits are particularly informative and might suggest what constitutes the nonoverlapping regions. Unfortunately, because the lesions in the neuropsychological patients are rather large, one cannot determine precise anatomical areas for these nonoverlapping regions, but insights into the behaviors selectively associated with imagery or perception have been obtained, as discussed next.

Patients with impaired imagery but intact perception are unable to draw or describe objects from memory, to dream, or to verify propositions based on memory ("does the letter $W$ have three strokes?"). It has been suggested that in these cases, the process of imagery generation (which does not overlap with perception) may be selectively affected without any adverse consequences for recogni- 
tion. I review this generation process in further detail in a later section. It has also been suggested that low- or intermediate-level processes may play a greater (but not exclusive) role in perception than they do in imagery. For example, when asked to image a "kangaroo," one accesses the intact longterm representation of a kangaroo, and this is then instantiated and available for inspection. When one is perceiving a kangaroo, however, featural analysis, as well as perceptual organization such as figureground segregation and feature grouping or integration, is required. If a patient has a perceptual deficit because of damage to these low- or intermediate-level processes, the patient will be unable to perceive the display, but imagery might well be spared because it relies less on these very processes (Behrmann, Moscovitch, \& Winocur, 1994).

\section{Summary and Challenges}

There is substantial evidence that imagery and perception share many (although not all) psychological and neural mechanisms. However, some results suggest that the early visual regions in the occipital lobe are not part of the shared network. For example, it has been shown that reliable occipital activation is not always observed in neuroimaging studies when subjects carry out mental imagery tasks. A possible explanation for these null results concerns the task demands: When the task does not require that the subjects form a highly depictive image, no occipital activation is obtained. A second explanation might have to do with subject sampling: There are considerable individual differences in mental imagery ability, and subjects who score poorly on a mental imagery vividness questionnaire show less blood flow in area 17 than those who score higher. If a study includes only low-imagery individuals, no occipital lobe activation might be obtained. A final explanation concerns the nature of the baseline, or control, condition used in neuroimaging experiments: If subjects are instructed to rest but instead continue to activate internal representations, when the activation obtained in the baseline is subtracted from that obtained in the imagery condition, no primary visual cortex activation will be observed.

Another challenge to the conclusion that primary visual areas are involved in imagery comes from studies of patients who have bilateral occipital lesions and complete cortical blindness but preserved imagery. Indeed, some of these patients are capable of generating such vivid images that they believe these to be veridical perceptions. For example, when a set of keys was held up before one such subject, she correctly identified the stimulus based on the auditory signal but then went on to provide an elaborate visual description of the keys, convinced that she could actually see them (Goldenberg, Müllbacher, \& Nowak, 1995). Another subject with bilateral occipital lesions whose perception was so impaired that he could not even differentiate light from dark was still able to draw well from memory and performed exceptionally well on standard imagery tasks (Chatterjee \& Southwood, 1995).

In sum, although the data supporting a strong association between imagery and perception are compelling, some findings are not entirely consistent with this conclusion.

\section{GENERATION OF MENTAL IMAGES}

A second major debate in the imagery literature concerns the mechanisms involved in generating a mental image. This is often assumed to be a process specific to imagery (or perhaps more involved in imagery than in perception) and involves the active construction of a long-term mental representation. Although there has been debate concerning whether there is such a process at all, several lines of evidence appear to support its existence and role in imagery. There are, for example, reports of neuropsychological patients who have preserved perception but impaired imagery and whose deficit is attributed to image generation. Patient R.M., for example, could copy well and make good shape discriminations of visually presented objects but could not draw even simple shapes nor complete from memory visually presented shapes that were partially complete (Farah, Levine, \& Calvanio, 1988).

One controversial and unanswered issue concerns the neural substrate of the generation process. The growing neuropsychological literature has confirmed the preponderance of imagery generation deficits in patients with lesions affecting the left temporo-occipital lobe regions. There is not, however, a perfect relationship between this brain region and imagery generation, as many patients with such lesions do not have an impairment in imagery generation.

In many studies, normal subjects show a left-hemisphere advantage for imagery generation; when asked to image half of an object, subjects are more likely to image the right half, reflecting greater left-hemisphere than righthemisphere participation in imagery generation. Additionally, righthanded subjects show a greater decrement in tapping with their right than left hand while performing a concurrent imagery task, reflecting the interference encountered by the left hemisphere while 
tapping and imaging simultaneously. (The left hemisphere controls movement on the right side of the body.) Studies in which information is presented selectively to one visual field (and thereby one hemisphere) have, however, yielded more variable results with normal subjects. Some studies support a left-hemisphere superiority, some support a right-hemisphere superiority, and some find no hemispheric differences at all. Studies with split-brain patients ${ }^{4}$ also reveal a trend toward lefthemisphere involvement, but also some variability. Across a set of these rather rare patients, imagery performance is better when the stimulus is presented to the left than to the right hemisphere, although this finding does not hold for every experiment and the results are somewhat variable even within a single subject.

Neuroimaging studies in normal subjects have also provided some support for a left-hemisphere basis for imagery generation. For example, functional magnetic resonance imaging showed more activation of the left inferior occipitaltemporal region when subjects generated images of heard words compared with when they were simply listening to these words (D'Esposito et al., 1997). This result had also been observed previously using $\mathrm{ERPs}^{5}$; an asymmetry in the waveforms of the two hemispheres implicated the left temporooccipital regions in imagery generation (see Farah, 1999).

In sum, there is a slight but not overwhelming preponderance of evidence favoring the left hemisphere as mediating the imagery generation process. A conservative conclusion from these studies suggests that there may well be some degree of left-hemisphere specialization, but that many individuals have some capability for imagery generation by the right hemisphere. Another suggestion is that both hemispheres are capable of imagery generation, albeit in different ways; for example, subjects showed a left-hemisphere advantage in a generation task when they memorized how the parts of a stimulus were arranged but showed a right-hemisphere advantage when they memorized the metric positions of the parts and how they could be "mentally glued" together (see Kosslyn, 1994).

\section{CONCLUSION}

Although considerable progress has been made in analyzing the convergence between imagery and perception, there are several outstanding issues. One of these is the relationship between imagery and the activation of internal representations in other cognitive tasks. For example, during visual working memory tasks, a mental representation of an object or spatial location is maintained over a delay period, in the absence of retinal stimulation. In these tasks, areas in the very front of the brain, rather than occipital cortex, are activated despite the similarities between this task and mental imagery. Similarly, in tasks that involve topdown forms of attention, subjects are verbally instructed to search for a target (such as a red triangle) in an upcoming display. Although subjects likely generate an image of a red triangle, this is generally not conceived of as an instance of mental imagery, and in neuroimaging studies the location of activation is not usually sought in occipital cortex.

Another perplexing and unresolved issue concerns the reasons that vivid imagery and hallucinations are not confused with reality, especially given that functional imaging studies show identical activations during hallucinations and perception (ffytche et al., 1998). Several solutions to this dilemma have been proposed, among them the idea that hallucinations derive from a failure to self-monitor an inner voice, with the result that the source of the stimulus is located in the external world. A second explanation suggests that perceptions are deeper and contain more detail than images. As Hume (1739/1963) stated, "The difference betwixt these [imagery-ideas and perception] consists in the degree of force and liveliness, with which they strike upon the mind .... Perceptions enter with most force and violence ... . By ideas I mean the faint images of these in thinking and reasoning" (p. 311). How to verify these claims empirically is not obvious, yet this issue clearly demands resolution.

\section{Recommended Reading}

Behrmann, M., Moscovitch, M., \& Winocur, G. (1999). Visual mental imagery. In G.W. Humphreys (Ed.), Case studies in vision (pp. 81-110). London: Psychology Press.

Farah, M.J. (1999). (See References)

Kosslyn, S.M. (1994). (See References)

Richardson, J.T.E. (1999). Imagery. Philadelphia: Psychology Press.

Acknowledgments-This work was supported by grants from the National Institute of Mental Health (MH54246 and MH54766). I thank Martha Farah and Steven Kosslyn for helpful discussions about mental imagery and Nancy Kanwisher for her insightful comments on this manuscript.

\section{Notes}

1. Address correspondence to Marlene Behrmann, Department of Psychology, Carnegie Mellon University, Pittsburgh, PA 15213-3890; e-mail: behrmann+@cmu.edu.

2. Visual information is received initially via the retina of the eye and is then transmitted through various visual pathways to the brain. This infor- 
mation is sent initially to the primary visual area of the brain, housed posteriorly in the occipital cortex, and is then sent more anteriorly through secondary visual areas to the temporal lobe of the brain for the purposes of recognition. The primary visual area is also known as area 17 or V1, and the secondary area is known as area 18 or V2. The visual input is also sent from the occipital cortex up to the parietal areas of the brain, which represent and code spatial information.

3. Repetitive transcranial magnetic stimulation is a new method in which magnetic pulses are delivered to the brain from a magnet placed externally on the scalp. The electrical pulses disrupt the function of the underlying brain area temporarily and are thus analogous to a reversible lesion. This method allows investigators to determine the involvement of certain brain areas in particular cognitive processes.

4. Split-brain patients are individuals who have undergone a separation of the two sides of the brain (cerebral hemispheres). This is done in individuals who have intractable and uncontrolled epilepsy in order to prevent the seizure activity from spreading across the entire brain. Unfortunately, it also prevents the transfer of all other forms of information from one hemisphere to the other.

5. ERPs, or evoked response poten- tials, are recordings of the brain's electrical activity in response to stimuli that are presented to the subject. The potentials are measured over time as waveforms obtained from electrodes placed at specific sites on the scalp, and different waveforms roughly reflect differential participation of some brain sites in the task under examination.

\section{References}

Behrmann, M., Moscovitch, M., \& Winocur, G. (1994). Intact visual imagery and impaired visual perception in a patient with visual agnosia. Journal of Experimental Psychology: Human Perception and Performance, 20, 1068-1087.

Chatterjee, A., \& Southwood, M.H. (1995). Cortical blindness and visual imagery. Neurology, 45, 2189-2195.

D'Esposito, M., Detre, J.A., Aguirre, G.K., Stallcup, M., Alsop, D.C., Tippett, L.J., \& Farah, M.J. (1997). Functional MRI study of mental image generation. Neuropsychologia, 35, 725-730.

Farah, M.J. (1999). Mental imagery. In M. Gazzaniga (Ed.), The cognitive neurosciences (Vol. 2, pp. 965-974). Cambridge, MA: MIT Press.

Farah, M.J., Levine, D.N., \& Calvanio, R. (1988). A case study of a mental imagery deficit. Brain and Cognition, 8, 147-164.

ffytche, D.H., Howard, R.J., Brammer, M.J., David, A., Woodruff, P., \& Williams, S. (1998). The anatomy of conscious vision: An fMRI study of visual hallucinations. Nature Neuroscience, 1 , 738-742.

Ghaem, O., Mellet, E., Crivello, F., Tzourio, N., Mazoyer, B., Berthoz, A., \& Denis, M. (1997). Mental navigation along memorized routes activates the hippocampus, precuneus and insula. NeuroReport, 8, 739-744.
Goebel, R., Khorram-Sefat, D., Muckli, L., Hacker, H., \& Singer, W. (1998). The constructive nature of vision: Direct evidence from functional magnetic resonance imaging studies of apparent motion and motion imagery. European Journal of Neuroscience, 10, 1563-1573.

Goldenberg, G., Müllbacher, W., \& Nowak, A (1995). Imagery without perception-A case study of anosognosia for cortical blindness. Neuropsychologia, 33, 39-48.

Howard, R.J., ffytche, D.H., Barnes, J., McKeefry, D., Ha, Y., Woodruff, P.W., Bullmore, E.T. Simmons, A., Williams, S.C.R., David, A.S., \& Brammer, M. (1998). The functional anatomy of imagining and perceiving colour. NeuroReport, 9, 1019-1023.

Hume, D. (1963). A treatise of human nature. In V.C. Chappel (Ed.), The philosophy of David Hume (pp. 11-311). New York: Modern Library. (Original work published 1739)

Kosslyn, S.M. (1994). Image and brain. Cambridge, MA: MIT Press.

Kosslyn, S.M., Pascual-Leone, A., Felician, O., Camposano, S., Keenan, J.P., Thompson, W.L., Ganis, G., Sukel, K.E., \& Alpert, N.M. (1999). The role of Area 17 in visual imagery: Convergent evidence from PET and TMS. Science, 284, 167-170.

Kosslyn, S.M., Thompson, W.L., \& Alpert, N.M (1997). Neural systems shared by visual imagery and visual perception: A positron emission tomography study. Neuroimage, 6, 320-334.

O'Craven, K., \& Kanwisher, N. (in press). Mental imagery of faces and places activates corresponding stimulus-specific brain regions. Journal of Cognitive Neuroscience.

Paivio, A. (1979). Imagery and verbal processes. Hillsdale, NJ: Erlbaum.

Pylyshyn, Z.W. (1981). The imagery debate: Analogue media versus tacit knowledge. Psychological Review, 88, 16-45.

Shepard, R.N., \& Cooper, L.A. (1982). Mental images and their transformations. Cambridge, MA: MIT Press. 